\title{
SÍNDROME DE GRADENIGO, UNA COMPLICACIÓN POCO FRECUENTE EN EL ADULTO. REPORTE DE UN CASO
}

\section{GRADENIGO SYNDROME, A RARE COMPLICATION IN ADULTS. CASE REPORT}

Juan Á. González-Barrón ${ }^{1 *}$ y Claudia A. Guerra-Gómez²

${ }^{1}$ Departamento de Urgencias; ${ }^{2}$ Departamento de Otorrinolaringología. Hospital General de Zona 11, Instituto Mexicano del Seguro Social, Nuevo Laredo, Tamaulipas, México

RESUMEN: El síndrome de Gradenigo es una complicación evolutiva de la otitis media supurativa. Fue descrito por primera vez por Giuseppe Gradenigo en 1904, presentándose la triada característica: otitis media aguda, neuralgia del trigémino y parálisis del VI par craneal. Afecta comúnmente a la edad pediátrica, así como a pacientes con inmunosupresión, diabetes mellitus, etc. Se atribuye a una petrositis apical aguda, complicación de una infección del oído medio. La sospecha clínica y los estudios de imagen, como la tomografía computarizada, nos confirman el diagnóstico. Actualmente el tratamiento de primera línea son antibióticos, pudiendo ser necesaria la intervención quirúrgica o ambos. La intención de este caso es saber reconocer oportunamente las manifestaciones clínicas y sensibilizar al personal de salud para su abordaje, diagnóstico y tratamiento. Presentamos el caso de un hombre de 76 años de edad, referido por presentar otitis externa bilateral de dos meses de evolución, con aparente mejoría, agregándose otorrea izquierda intermitente, parálisis del VI par craneal derecho, así como otalgia y dolor retroocular ipsilateral.

Palabras clave: Síndrome de Gradenigo. Otitis media. Petrositis.
ABSTRACT: Gradenigo syndrome is an evolutionary complication of suppurative otitis media, first described by Guiseppe Gradenigo in 1907, presenting the characteristic triad: acute otitis media, trigeminal neuralgia and VI of the cranial nerve. It commonly affects pediatric age as well as patients with immunosuppression, diabetes mellitus, etc. It is attributed to an acute apical petrositis, complication of a middle ear infection. Clinical suspicion and imaging studies such as computed tomography (CT) confirm the diagnosis. Currently the treatment is antibiotics, and surgery or both may be necessary. The intention of this case is to know how to recognize the clinical manifestations in a timely manner and sensitize the health personnel for their diagnostic and therapeutic approach. We present the case of a 76-year-old male, referred for presenting bilateral otitis externa of 2 months evolution, with apparent improvement, adding intermittent left otorrhea, paralysis of the VI right cranial nerve as well as otalgia and ipsilateral retro ocular pain.

Key words: Gradenigo syndrome. Otitis media. Petrositis.
Correspondencia:

*Juan Á. González-Barrón

E-mail: drangelglzb@ hotmail.com
Fecha de recepción: 01-12-2020

Fecha de aceptación: 29-04-2021
Disponible en internet: 03-12-2021

Rev Mex Med Fam. 2021;8:97-101 DOI: 10.24875/RMF.20000186

2007-9710 / @ 2021 Federación Mexicana de Especialistas y Residentes en Medicina Familiar. Publicado por Permanyer. Este es un artículo open access bajo la licencia CC BY-NC-ND (http://creativecommons.org/licenses/by-nc-nd/4.0/). 


\section{HISTORIA}

En una publicación realizada a la Real Academia de Medicina de Turín en 1904, Gradenigo, un médico italiano, presentó por primera vez algunos casos de parálisis del nervio motor ocular externo de origen postinfeccioso del oído medio, formando la base para la descripción de un síndrome actualmente conocido como síndrome de Gradenigo ${ }^{1}$.

\section{INTRODUCCIÓN}

Las complicaciones de la otitis media desde el empleo de antimicrobianos son relativamente infrecuentes. Se han clasificado tradicionalmente en intratemporales (mastoiditis, petrositis, laberintitis, parálisis facial) e intracraneales (meningitis, absceso subdural, extradural o cerebral, tromboflebitis de los senos venosos e hipertensión intracraneal benigna).

El síndrome de Gradenigo se caracteriza por dolor facial en la zona inervada por el trigémino y parálisis del VI par craneal ipsilateral secundaria a petrositis apical aguda, por complicación evolutiva de una otitis media ${ }^{2}$.

La fisiopatología de este síndrome es la inflamación local de la duramadre adyacente a la porción petrosa del hueso temporal, comprometiendo al V y VI par craneal. Considera la porción petrosa del hueso temporal como una pirámide de base externa, el proceso inflamatorio se extiende desde la base del oído medio y la mastoides hacia el extremo medial por contigüidad. El tratamiento de la petrositis aguda ha evolucionado desde la cirugía radical en la era preantibiótica, al tratamiento médico y cirugía menor en los últimos años ${ }^{3}$.

Aunque es raro, este síndrome puede tener complicaciones incluso mortales, siendo algunas de estas complicaciones: meningitis, trombosis del seno lateral, absceso epidural, empiema subdural, encefalitis focal ótica, absceso extradural, laberintitis y mastoiditis crónica.
Afecta principalmente a los pacientes con exposición a dosis altas de esteroides. Los pacientes diabéticos son particularmente susceptibles a padecer otitis media supurada y otitis externa maligna ${ }^{4}$. La oftalmoplejía externa y la diplopía concomitante de comienzo más o menos agudo plantea los diagnósticos diferenciales de las siguientes entidades clínicas: traumatismos, neoplasias, hipertensión endocraneal y procesos infecciosos intracraneales tales como trombosis del seno venoso lateral, y abscesos intracraneales epidurales y subdurales ${ }^{5}$. En un estudio realizado por el Departamento de Otorrinolaringología de la Universidad Médica del Norte de New York SUNY (Upstate Medical University SUNY) en 2018, se encontró que la edad promedio de aparición es de 22.4 años de edad, la distribución entre hombre y mujer es igual, y la mortalidad es del $2.6 \%$ de los casos. También se revisaron los tratamientos utilizados en 37 pacientes a lo largo de la historia del síndrome de Gradenigo desde 1990 hasta la actualidad encontrando que el tratamiento fue más o menos uniforme distribuido entre tratamiento antibiótico solo y combinado con intervención quirúrgica. Catorce de 37 pacientes fueron tratados con antibióticos por vía intravenosa (IV) solamente. Los tratamientos quirúrgicos junto con la terapia antibiótica IV varió desde simples tubos de timpanostomía en seis pacientes, a mastoidectomía sola en tres pacientes, mastoidectomía combinada con extracción del hueso del ápice petroso en dos pacientes, mastoidectomía combinada con craneotomía temporal en dos pacientes, y combinada subtemporal y craneotomía de la fosa media junto con radiación postoperatoria terapia en un paciente. Esto demuestra que la agresividad del tratamiento quirúrgico debe adaptarse al curso clínico, factores comórbidos o precipitantes, y hallazgos radiográficos en cada caso. El síndrome de Gradenigo debe mantenerse en el diagnóstico diferencial para pacientes con infecciones otológicas, particularmente si la parálisis del sexto nervio está presente. Las imágenes 
con tomografía computarizada (TC) o resonancia magnética (RM) deben ser utilizadas para confirmar los hallazgos clínicos y evaluar los asociados a malignidad o absceso, y tratamiento antibiótico IV agresivo combinado con cirugía cuando sea necesario se debe implementar tan pronto como sea posible ${ }^{6}$.

En los estudios de imagen la TC muestra evidencia de opacidad en las células de aire a nivel del conducto petromastoideo, posiblemente destrucción ósea, y es particularmente útil en diagnóstico de evidencia de la formación de un absceso intracraneal. La RM muestra una inflamación en la porción petrosa ${ }^{7}$.

Hasta el momento no hemos encontrado referencias bibliográficas actuales o datos actualizados del síndrome de Gradenigo en nuestro país, por lo que consideramos de suma importancia para el personal de primer nivel identificar a tiempo los signos y síntomas, así como su manejo oportuno.

\section{CASO CLÍNICO}

Paciente de sexo masculino de 76 años de edad, con antecedentes personales patológicos: diabetes mellitus tipo II e hipertensión arterial sistémica de más de 15 años de evolución con mal control, con secuelas microvasculares y macrovasculares. Antecedentes quirúrgicos: amputación bilateral supracondílea hace seis años por pie diabético. Alergias negadas. Inicia su padecimiento actual hace dos meses con otalgia y otorrea derecha, hipoacusia, acúfenos de tonos agudos ipsilaterales, así como cefalea occipital ipsilateral, acudiendo a médico particular y siendo tratado con antibioticoterapia vía oral y tópica (se desconoce tratamiento), sin respuesta favorable. Al continuar sintomatología y agregándose dolor en hemicara derecha, refiriendo en una escala visual analógica (EVA) 6/10, automedicándose con ketorolaco $30 \mathrm{mg}$ intramuscular cada $12 \mathrm{~h}$ y diclofenaco $100 \mathrm{mg}$ vía oral (VO) cada 8 h, sin mejoría.

A su ingreso el paciente se encontraba con los siguientes signos vitales: tensión arterial 185/94 mmHg, frecuencia cardiaca 105 latidos por minuto, frecuencia respiratoria 18 respiraciones por minuto, temperatura $36^{\circ} \mathrm{C}$, glucosa capilar $250 \mathrm{mg} / \mathrm{dl}$; en actitud hipoacúsica, alerta, cooperador, con facies de dolor, hidratado, sin compromiso de pares craneales, dolor en hemicara derecha sin edema. En la otoscopia derecha se encontró detritus y otorrea que no permitía la visualización completa de la membrana timpánica, se procede a aspiración. Rinoscopia: ambas fosas nasales permeables, septum funcional, cornetes en ciclo y sin descarga retronasal. Tórax simétrico con buena entrada y salida del aire, no se integra en este momento síndrome pleuropulmonar, abdomen blando depresible globoso a expensas de tejido adiposo, timpanismo en todo marco cólico, peristalsis presente, sin datos de abdomen agudo en este momento, amputación supracondílea bilateral. Se decide a instaurar tratamiento tópico a base de ciprofloxacino, lidocaína e hidrocortisona, y tratamiento $\mathrm{VO}$ con ciprofloxacino 500 mg cada $12 \mathrm{~h}$, así como control de glucemia, y se insiste y explica el cuidado del oído contra el agua. Se cita en cinco días para valoración. Acude a su cita de valoración y se refiere disminución de otorrea, persistiendo dolor en hemicara derecha, sin embargo, lo refiere en menor intensidad (EVA 4/10). A la otoscopia se observa conducto auditivo externo derecho húmedo, aún sin observar la membrana timpánica en su totalidad por edema, pares craneales conservados; resto de la exploración física normal. Se insiste en el control glucémico y continuar con tratamiento y cuidados instaurados, y se cita para nueva valoración en una semana.

El paciente no acude a su cita programada, acudiendo tres semanas posteriores, refiriendo que suspendió tratamiento por mejoría de dolor, inicia nuevamente hace tres días con otorrea y otalgia derecha, así como aumento de dolor (EVA 8/10) en hemicara derecha. A la exploración física se encuentra con parálisis del VI (motor ocular externo) par craneal derecho (Fig. $1)$, resto de pares craneales respetados. A 


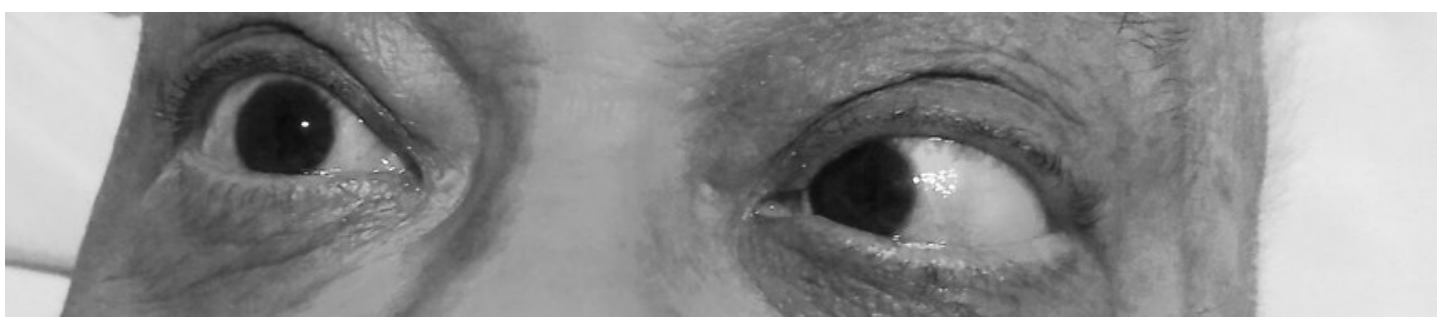

Figura 1. Estrabismo divergente del ojo derecho secundario a parálisis del VI par craneal.

la otoscopia derecha se observa abundante otorrea y detritus, así como edema del conducto auditivo externo en su tercio medio y externo, sin permitir visualización de membrana timpánica, con dolor a la palpación de región mastoidea, por lo que se decide su ingreso a este hospital, para iniciar protocolo de estudio. Se solicita interconsulta al servicio de medicina interna para control metabólico. Se realiza TC, en la cual se observa mastoides izquierda bien neumatizada y con celdillas libres. Mastoides derecha con celdillas mastoideas ocupadas en su totalidad por densidad de tejidos blandos, extendiéndose hacia el peñasco del temporal con aparente lisis ósea (Fig. 2), confirmando la sospecha clínica de síndrome de Gradenigo derecho.

Signos vitales: tensión arterial 180/89 $\mathrm{mmHg}$, frecuencia cardiaca 107 latidos por minuto, frecuencia respiratoria 20 respiraciones por minuto, temperatura $36.3^{\circ}$. Laboratorio: en parámetros normales.

Evolución clínica: el paciente permanece hospitalizado por 20 días con mejoría clínica a los cinco días de dolor, remitiendo otorrea, persistiendo parálisis del VI par craneal. El tratamiento instaurado fue ceftriaxona $1 \mathrm{~g}$ IV cada $12 \mathrm{~h}$ y clindamicina 600 mg IV cada 8 h y gotas óticas a base de ciprofloxacino, lidocaína e hidrocortisona, así como cuidado estricto de oído contra el agua, y limpieza y aspiración diaria de oído. El servicio de medicina interna controló las glucemias séricas. El paciente al notar mejoría decide su alta voluntaria, se manejó en casa con antibioticoterapia vía oral, moxifloxacino $400 \mathrm{mg}$ VO cada 24 h, gotas óticas antes mencionadas y cuidados de oído

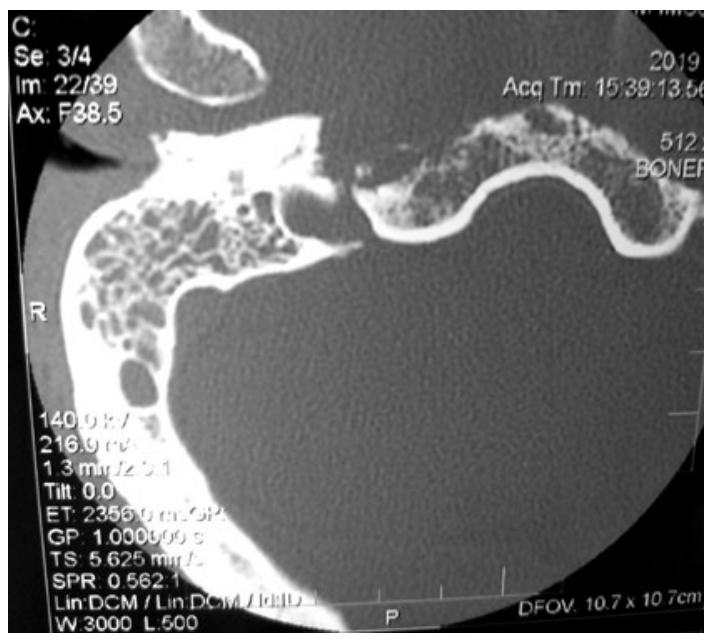

Figura 2. Tomografía axial computarizada de mastoide derecha.

contra el agua; se dan datos de alarma y se cita a valoración en cinco días.

\section{DISCUSIÓN}

Se trata de un paciente de 76 años con factores de riesgo como la hipertensión arterial sistémica y diabetes mellitus 2 con mal control y complicaciones microvasculares y macrovasculares, otitis media, tratada de forma tardía y con mal apego al tratamiento y cuidados de oído, instalándose la parálisis del VI par craneal derecho a las 12 semanas de iniciar con síntomas otológicos, exacerbándose el dolor en hemicara derecha y la diplopía concomitante. En el 30\% de los individuos el hueso del ápice petroso está constituido por celdillas aereadas que comunican con la cavidad del oído medio.

El intervalo de tiempo entre el proceso supurativo del oído medio y el involucro del ápex petroso es de 1-12 semanas, en nuestro caso clínico pasaron 12 semanas desde 
el inicio de la otitis media hasta la parálisis del VI par y la neuralgia del trigémino apareció a las cuatro semanas. Los hallazgos típicos en la TC son: ocupación de las celdillas mastoideas por material del oído medio, destrucción ósea, gas en el seno dural y formación de abscesos intracraneales o extradurales. Al paciente se le indicó la realización de una $\mathrm{TC}$, en la cual se observaron celdillas mastoideas ocupadas, en su totalidad, así como también celdillas de área petrosa con lisis de algunas de estas; no se observaron abscesos intracraneales o extracraneales.

En el caso presentado se inició un tratamiento agresivo en la consulta externa con antibióticos tanto tópicos como orales, con mejoría parcial, a su revaloración a las tres semanas (donde el paciente suspendió medicamento y cuidados por mejoría) se instauró la parálisis del VI par craneal derecho y se exacerbó el dolor, por lo que se decide su manejo hospitalario. Durante su hospitalización mejoraron los síntomas a partir del 5. ${ }^{\circ}$ día, suspendiendo tratamiento intrahospitalario por alta voluntaria del paciente al notar mejoría.

\section{FINANCIAMIENTO}

La presente investigación no ha recibido ninguna beca específica de agencias de los sectores públicos, comercial o sin ánimo de lucro.

\section{CONFLICTO DE INTERESES}

Los autores declaran no tener conflicto de intereses.

\section{RESPONSABILIDADESÉTICAS}

Protección de personas y animales. Los autores declaran que para esta investigación no se han realizado experimentos en seres humanos ni en animales.

Confidencialidad de los datos. Los autores declaran que han seguido los protocolos de su centro de trabajo sobre la publicación de datos de pacientes.

Derecho a la privacidad y consentimiento informado. Los autores han obtenido el consentimiento informado de los pacientes y/o sujetos referidos en el artículo.

\section{BIBLIOGRAFÍA}

1. Matis GK, de A Silva DO, Chrysou OI, Karanikas MA, Birbilis TA Giuseppe Gradenigo: Much more than a syndrome! Historical vignette. Surg Neurol Int. 2012;3:122.

2. Pelegrín-Hernández JP, Díaz-Manzano JA, MenasalvasRuiz Al, Hellín-Meseguer D. Síndrome de Gradenigo y trombosis de seno cavernoso secundaria a otitis media aguda. Rev Soc Otorrinolaringol Castilla Leon Cantab La Rioja. 2012;3(10):99 106.

3. Methol G, García L, Giachetto G. Síndrome de Gradenigo: Una complicación poco frecuente de la otitis media aguda. Arch Pediatr Urug. 2016;87(1):44-8.

4. Ordoñez Mejía ES. Síndrome de Gradenigo: Presentación de un caso. Rev Fac Cienc Med. 2011;8:29-34.

5. Neipp López R, Vizcaíno Díaz C, Revert Lázaro F, Espinosa Seguí N, Fuentes Castelló MA, Neipp Lindenau C. Oftalmoplejia externa secundaria a petrositis apical: Síndrome de Gradenigo. Rev Pediatr Aten Primaria. 2003;5:571-5.

6. Gore MR. Gradenigo's syndrome: A Review. Ann Med Health Sci Res. 2018;8:220-4.

7. Murakami T, Tsubaki J, Tahara Y, Nagashima T. Gradenigo's syndrome: CT and MRI findings. Pediatr Radiol. 1996;26:684-5. 